\title{
Fisioterapia y la ley del medicamento y productos sanitarios: el triunfo de la sin razón
}

\author{
Physiotherapy and the law of medicines and medical devices: the triumph of \\ unreasonableness
}

\section{González Doniz}

Escuela Universitaria de Fisioterapia y Departamento de Fisioterapia Universidad de A Coruña, A Coruña, España

Hace unos meses se debatió y aprobó en el Parlamento proposición de ley de modificación de la Ley 29/2006, de 26 de julio, de garantías y uso racional de los medicamentos y productos sanitarios. Resulta muy ilustrativa la lectura del borrador del pleno del Senado en el que se abordó este tema, de gran importancia para los profesionales sanitarios y con, como todos sabemos, un resultado negativo para las legítimas aspiraciones de los fisioterapeutas, al quedar excluidos del grupo de profesionales con capacidad para prescribir medicamentos y productos sanitarios. Digo que resulta ilustrativo por el "peso" y "contundencia" de los argumentos en los que algunas de sus señorías basaron nuestra exclusión. Entre ellos cabe destacar lo que sigue: "Comprendemos, evidentemente, que otros profesionales presenten demandas para alcanzar dichas responsabilidades, pero he de comunicarles, Señorías, que dichas peticiones, en nuestra opinión, no presentan criterios de calidad de formación ni de capacitación para dichas responsabilidades de las que estamos hablando, en sus formaciones académicas respectivas" basando esta ausencia de criterios de calidad en que "en los planes de formación de los fisioterapeutas no tienen recogida la adquisición de conocimientos para desarrollar esa responsabilidad de prescripción. Hace tiempo, los fisioterapeutas venían como una especialidad de enfermería y, en este caso sí la tendrían, pero para los que entran por las escuelas actuales, la situación actual es que no en todas las escuelas se recibe formación de farmacología y patología clínica, que son necesarias y evidentes para dispensar medicamentos. Igualmente, en la sanidad pública, en los hospitales, los fisioterapeutas no son autónomos y su trabajo les es remitido por parte de los profesionales médicos o de enfermería, que son los que hacen la revisión que ha hecho el fisioterapeuta".

Es lamentable el desconocimiento de la fisioterapia de quien así se expresa, máxime si proviene, como es el caso, del ámbito sanitario. En primer lugar, en el Real Decreto 1414/1990 sobre directrices generales propias de los planes de estudio del título oficial de fisioterapia, que regula los títulos de diplomado en fisioterapia, se recoge como materia troncal las afecciones médicas y afecciones quirúrgicas y sus tratamientos: aspectos generales de la patología de origen externo e interno de todos los aparatos y sistemas, con sus tratamientos médicos, quirúrgicos, fisioterápicos y ortopédicos. Asimismo, la Orden CIN/2135/2008, de 3 de julio, por la que se establecen los requisitos para la verificación de los títulos universitarios oficiales que habiliten para el ejercicio de la profesión de fisioterapeuta, incluye, entre otros, el siguiente contenido formativo común (recordamos, de obligada inclusión en todos los planes de estudio de todas las universidades): "Conocer la fisiopatología de las enfermedades identificando las manifestaciones que aparecen a lo largo del proceso, así como los tratamientos médicos y quirúrgicos, fundamentalmente en los aspectos fisioterapéuticos y ortopédicos".

En la regulación académica queda pues claramente recogida la obligatoriedad del estudio de la patología y sus tratamientos médicos. ¿A qué tratamientos médicos puede referirse, entre otros, la citada regulación si no es a los farmacológicos?

La reforma de la citada ley del medicamento tiene su fundamento, y así se recoge en la misma, en la ley de ordenación de las profesiones sanitarias que establece como función de los fisioterapeutas "la prestación de los cuidados propios de su disciplina, a través de tratamientos con medios y agentes físicos, dirigidos a la recuperación y rehabilitación de personas con disfunciones o discapacidades somáticas, así 
como a la prevención de las mismas". Parece que hay que insistir, una vez más, en que la fisioterapia trata (tratamiento con agentes y medios físicos, con el objetivo de recuperar...) y la fase inicial de ese proceso es la prescripción. Los miles de fisioterapeutas que ejercen su actividad asistencial en el ámbito público y privado (mayoritariamente en este último) efectúan un diagnóstico fisioterápico y prescriben agentes físicos basándose en el razonamiento clínico adquirido, durante su formación de grado, a través de una exigente formación teórica, práctica y clínica. Es, por tanto, la prescripción un acto intrínseco de la fisioterapia, en absoluto ajena a los profesionales que la desarrollan.

La realidad de la situación de la fisioterapia en la sanidad pública es muy diferente según la comunidad autónoma (no han de olvidar sus señorías, que las competencias en materia sanitaria han sido transferidas a estas administraciones), el nivel asistencial de que se trate, la organización interna de cada hospital, así como de los servicios en los que se preste la asistencia. Pero lo más importante, la fisioterapia, a diferencia de la enfermería o la medicina, desarrolla, mayoritariamente, su actividad en el ámbito privado (al igual que los odontólogos y podólogos), situación compartida con todos los países desarrollados, con toda autonomía. Regularla tomando como referencia, exclusivamente, la sanidad pública constituye un error de grandes dimensiones ya que no se responde a su realidad actual, sino a la obsoleta realidad de hace 30 años.

Resulta especialmente grave que los fisioterapeutas no puedan prescribir medicamentos y productos sanitarios relacionados con su especialidad, cuando esos mismos productos pueden ser adquiridos por los ciudadanos en las oficinas de farmacia sin más filtro que su capacidad económica para costearlos. Se niega la capacidad y la competencia de unos profesionales sanitarios cuando se pueden adquirir analgésicos, antibióticos, antiinflamatorios y un largo etcétera de medicamentos y productos sanitarios, no ya en las oficinas de farmacia, sino a través de Internet o a través de los medios de comunicación, sin que nuestros responsables públicos adopten ninguna medida para velar por la tan cacareada salud de los ciudadanos.

Pero la modificación de ley objeto de esta editorial no aborda exclusivamente la prescripción del medicamento. Regula, además, la prescripción de productos sanitarios. Y, en mi opinión, en este apartado nuestra exclusión adquiere una especial relevancia. La Ley 29/2006, de 26 de julio, de garantías y uso racional de los medicamentos y productos sanitarios define producto sanitario como cualquier instrumento, dispositivo, equipo, material u otro artículo, utilizado solo o en combinación, incluidos los programas informáticos que intervengan en su buen funcionamiento, destinado por el fabricante a ser utilizado en seres humanos con fines de:

1. Diagnóstico, prevención, control, tratamiento o alivio de una enfermedad.

2. Diagnóstico, control, tratamiento, alivio o compensación de una lesión o de una deficiencia.

3. Investigación, sustitución o modificación de la anatomía o de un proceso fisiológico.

4. Regulación de la concepción.

Y que no ejerza la acción principal que se desee obtener en el interior o en la superficie del cuerpo humano por medios farmacológicos, inmunológicos ni metabólicos, pero a cuya función puedan contribuir tales medios.

¿Cómo puede atribuírsele incompetencia a un profesional sanitario para prescribir los dispositivos, instrumentos, equipos, etc. destinados a los fines recogidos en la ley, propios de su disciplina? ¿Cómo puede atribuírsele incompetencia y falta de formación a los fisioterapeutas para prescribir un TENS, una férula para una postura osteoarticular, un dispositivo de entrenamiento de los músculos respiratorios, un espirómetro incentivador, una silla de ruedas, un andador, unas muletas, un parapodium, unos bitutores, material para vendajes funcionales, rodilleras, etc., etc., etc.? ¿Cómo puede atribuírsele incompetencia al fisioterapeuta para prescribir los productos sanitarios propios de su disciplina y necesarios para la aplicación de los agentes físicos que prescribe tales como el calor, el frío, la electricidad, etc. y de utilización diaria en la práctica clínica?

Todo ello está sucediendo en el siglo XXI en España, con miles de fisioterapeutas responsables de la gestión clínica de los centros de fisioterapia, con miles de fisioterapeutas especializados, con cientos de fisioterapeutas en la academia y en la gestión académica, con másteres oficiales, programas de doctorado, doctores e investigadores. Todo ello está sucediendo cuando estamos estrenando la instauración de los nuevos títulos de grado que reconocen entre las competencias la capacidad del fisioterapeuta para dirigir autónomamente la atención fisioterápica y realizar el diagnóstico fisioterápico, por cierto, ratificado por una reciente sentencia judicial. Todo ello es fruto del desconocimiento de unos y de las acciones de otros; aquellos que se resisten a que la fisioterapia siga avanzando. No lo han conseguido antes ni lo lograrán ahora si tenemos claras 